\title{
ANÁLISE EPIDEMIOLÓGICA DA EVOLUÇÃO DOS CASOS DE MENINGITE NO ESTADO DE SÃO PAULO, NO PERÍODO DE 2016 A 2020 EM CRIANÇAS E PRÉ-ADOLESCENTES
}

\section{EPIDEMIOLOGICAL ANALYSIS OF THE EVOLUTION OF MENINGITIS CASES IN THE STATE OF SÃO PAULO, FROM 2016 TO 2020 IN CHILDREN AND PRE- ADOLESCENTS}

\author{
Sanna Castro Tavares ${ }^{1}$ \\ Samara Andréa da Costa Fonseca ${ }^{2}$ \\ Iris Leite Gomes ${ }^{3}$ \\ Lívia Saraiva Carriconde 4 \\ Clara Cecília Rodrigues Mendes ${ }^{5}$ \\ Karla Khalil Menezes de Miranda ${ }^{6}$ \\ Verena Pamponet Magalhães ${ }^{7}$ \\ Carolina Takematsu ${ }^{8}$ \\ Kevyn Felipe Mendes ${ }^{9}$ \\ Marcos Arturo Ferreira Aguero ${ }^{10}$
}

RESUMO: Introdução: A meningite é uma inflamação que acomete as membranas do encéfalo tornando-se uma doença de grande morbidade e mortalidade. Ademais, a faixa etária mais acometida pela doença é a de crianças em idade escolar. Objetivo: Descrever o perfil epidemiológico dos casos de meningite, notificados e confirmados, entre as crianças de o a 12 anos e dos pré-adolescentes de 12 a 14 anos do estado de São Paulo, no período de 2016 a 2020, incluindo sexo e evolução, além de comparar os grupos etários analisados. Metodologia: Esse artigo refere-se a um estudo epidemiológico de caráter observacional, transversal, descritivo, com abordagem quantitativa e de prevalência, realizado através do Sistema de Informação de Agravos de Notificações (SINAN). Resultados: Foram analisados 15.332 casos de meningite. Logo, observou-se uma prevalência no ano de 2018, com 4.234 casos. Além disso, notou-se que a Meningite esteve mais presente na faixa etária de r a 4 anos e em indivíduos do sexo masculino. Ademais, foi observado que a taxa de altas foi de 96,39\% enquanto as taxas de óbito foram em apenas 2,9\% dos casos estudados. Discussão: Observou-se que a meningite foi frequente em indivíduos na faixa etária de i a 4 anos, devido a baixa imunidade dessa população. No entanto, notou-se que a maior parte dos agentes etiológicos da meningite pode ser evitada por meio da vacinação. Além disso, analisou-se que os indivíduos do sexo masculino representaram a maior porcentagem de mortalidade, sendo $56,4 \%$. Em contrapartida, houve uma alta taxa de liberação hospitalar, representada por

\footnotetext{
I Discente em Medicina - Universidade Estadual de Roraima (UERR). E-mail: sannactavares@gmail.com

${ }^{2}$ Discente em Medicina - UFAM. E-mail: samaradacostafonseca@gmail.com

3 Discente em Medicina - FAMESC BJI. E-mail: irisgomesi5.ig@gmail.com

${ }^{4}$ Discente em Medicina - UFPEL. E-mail: carriconde.livia@gmail.com

${ }^{5}$ Discente em Medicina - UNIRV.E-mail: claracecilia23II@gmail.com

${ }^{6}$ Discente em Medicina - UnirG. E-mail: karlakhalil23@gmail.com

7 Discente em Medicina - FAMESC BJI.E-mail: verena_pamponet@hotmail.com

${ }^{8}$ Discente em Medicina - FASM. E-mail: ctakematsuoficial@gmail.com

9 Discente em Medicina - Universidad Internacional Tres Fronteras. E-mail: kevynmedicina@gmail.com

ro Professor Doutor - Universidad Nacional de Asuncion. E-mail: ingeniero.ferreirar@gmail.com
} 
96, 39\% dos casos no período avaliado. Conclusão: A meningite é uma enfermidade que persiste na população de crianças e adolescentes no Estado de São Paulo, ocasionando uma diminuição na qualidade de vida, morbidade e a morte, em 2,9\% dos casos no período apresentado. Portanto, é fundamental que medidas profiláticas sejam incentivadas por parte das esferas governamentais em saúde e praticadas pela comunidade. Além disso, é necessário que se mantenha a notificação compulsória dessa doença por meio de atualizações epidemiológicas feitas de forma eficiente.

Palavras-chave: Meningite. Saúde pública.

ABSTRACT: Introduction: Meningitis is an inflammation that affects the brain membranes, making it a disease of great morbidity and mortality. Furthermore, the age group most affected by the disease is schoolage children. Objective: To describe the epidemiological profile of reported and confirmed cases of meningitis among children aged o to 12 years and pre-adolescents aged 12 to 14 in the state of São Paulo, in the period from 2016 to 2020, including sex and evolution, in addition to comparing the analyzed age groups. Methodology: This article refers to an observational, cross-sectional, descriptive epidemiological study with a quantitative and prevalence approach, carried out through the Notifiable Diseases Information System (SINAN). Results: 15,332 cases of meningitis were analyzed. Therefore, there was a prevalence in the year 2018, with 4,234 cases. In addition, it was noted that Meningitis was more present in the age group from $\mathrm{I}$ to 4 years and in males. Furthermore, it was observed that the discharge rate was $96.39 \%$ while death rates were only $2.9 \%$ of the cases studied. Discussion: It was observed that meningitis was frequent in individuals aged between I and 4 years, due to the low immunity of this population. However, it was noted that most etiologic agents of meningitis can be prevented through vaccination. In addition, it was analyzed that male individuals represented the highest percentage of mortality, being 56.4\%. On the other hand, there was a high rate of hospital discharge, represented by $96.39 \%$ of cases in the period evaluated. Conclusion: Meningitis is a disease that persists in the population of children and adolescents in the State of São Paulo, causing a decrease in quality of life, morbidity and death in $2.9 \%$ of cases in the period presented. Therefore, it is essential that prophylactic measures are encouraged by government health spheres and practiced by the community. In addition, it is necessary to maintain compulsory notification of this disease through epidemiological updates carried out efficiently.

Keywords: Meningitis. Public health.

\section{INTRODUÇÃO}

A meningite é uma inflamação que acomete as membranas que envolvem o encéfalo e a medula espinhal. Essa inflamação comumente é causada por vírus, bactérias, parasitas, fungos, mas também pode estar relacionada a cistos ou tumores intracerebrais, doenças inflamatórias e medicamentos (TAKADA et al., 2016).

Devido às suas altas taxas de morbimortalidade é considerada um problema de saúde pública, podendo desencadear, especialmente em crianças, importantes sequelas neurológicas e até mesmo a morte (OLIVEIRA; SIMÃO; NASCIMENTO, 2004). A meningite faz parte do rol de doenças de notificação compulsória imediata, fazendo com que os profissionais de saúde notifiquem os casos suspeitos ou confirmados em até 24 horas, e essas notificações devem ser registradas no Sistema de Informação de Agravos de Notificação - SINAN (BRASIL, 20I6). 
Em relação à sua etiologia, a meningite viral é a mais comum conforme a literatura. No entanto, a meningite bacteriana apresenta maior repercussão dentre as demais etiologias devido a sua alta taxa de morbidade e mortalidade (RODRIGUES; 2015). Em quantidade significativa de casos, os sinais e sintomas da meningite independem da etiologia, cursando com um quadro clínico clássico dessa doença como febre, cefaléia, vômitos, rigidez de nuca, sinal de Kernig. Os casos mais severos podem ainda evoluir para coma ou óbito (ROCHA; COSTA; SILVA, 2019).

O principal modo de transmissão da meningite são gotículas e secreções respiratórias, assim como há os casos de enterovírus em que a transmissão é fecal-oral, prevalecendo a infecção de pessoa a pessoa. Por conta disso, cidades com grande densidade populacional, como São Paulo, as habitações com muitos residentes e a translocação por transportes públicos lotados, podem contribuir para a propagação da doença (IVERSSON, 1976).

Ao tratar-se da faixa etária mais atingida pela meningite, ela é mais frequente em crianças em idade escolar, o que pode estar relacionado não somente com a fácil transmissão, mas também com o sistema imune em desenvolvimento nessa idade. Em relação à imunidade, deve-se levar em consideração que a defesa do organismo se desenvolve ao longo dos anos após o nascimento (CONDINO-NETO, 2014). Nesse sentido, é possível observar que as crianças possuem suscetibilidade para contrair infecções e, consequentemente, necessitam de mais doses de vacinas.

Ademais, na idade pré-escolar há o convívio em locais onde o contato com outras pessoas é significativo e as crianças não possuem o hábito de higiene, como lavar as mãos com frequência, por isso, se encontram tão vulneráveis (NESTI; GOLDBAUM, 2007).

A prevalência da meningite é de aproximadamente 2 casos a cada Ioo.00o habitantes, acometendo na maioria dos casos lactentes entre 3 e 12 meses, crianças menores de 5 anos, idosos com idade superior a 60 anos e pacientes imunocomprometidos. Pode também ser observada a elevação gradativa dos casos entre adolescentes e adultos jovens (EMMERICK et al., 2014).

A letalidade dela é bastante elevada (MAGALHÃES ; SANTOS, 2018), podendo variar entre 3 a 19\% (SANTOS et al., 202I). Nesse sentido, segundo dados observados da Organização Mundial da Saúde (OMS), estima-se que ocorram aproximadamente I,2 
milhão de casos e 135 mil mortes provocadas pela meningite anualmente no mundo (EMMERICK et al.,2014).

Diferentes fatores como as características socioeconômicas dos grupos, a existência de aglomerados populacionais, o meio ambiente, o agente infeccioso, a condição imunológica e a faixa etária do hospedeiro, interferem diretamente na expressão e prevalência epidemiológica da meningite a nível mundial (BRASIL, 2007).

No Brasil, nas décadas de 70 e 8o, foram identificadas epidemias em várias cidades do país (BRASIL, 2009). No município de São Paulo ocorreram entre 1920 e 2002 quatro epidemias de meningite. A primeira epidemia da doença meningocócica, deu-se em 1920, principalmente em razão das condições precárias de vida da população operária somadas ao agravamento da situação econômica e social dada pela ${ }^{\underline{a}}$ guerra mundial e aos impactos da gripe espanhola, normalizando-se em novembro de 1926 (MORAES; BARATA, 2005).

Os anos posteriores representaram um início insidioso da epidemia em SP. A segunda epidemia em SP, ocorreu entre 1945-1952, onde taxas de incidência que eram inferiores a 2 casos a cada Ioo mil habitantes, passaram abruptamente a quase 9 casos para cada Ioo.ooo habitantes, em 1945. O pico máximo foi em 1947, mas retornou aos valores endêmicos em 1952. A explosão de casos de meningite em SP ocorreu em 1974, caracterizando uma terceira epidemia, na qual apesar do início insidioso, houve então uma incidência alcançando a casa de 179,I por Ioo mil habitantes. A mortalidade acompanhou lentamente a incidência dos casos e atingiu seu pico em 1975. Nos anos consecutivos, observou-se declínio, voltando a aumentar a incidência e a ultrapassar o limiar endêmico em 1988, mostrando o surgimento de uma quarta epidemia, que atingiu níveis normais apenas em 2002 (MORAES; BARATA, 2005).

O estado de São Paulo é o estado com maior número de notificações de meningite (BRASIL, 2016). Dessa forma, vê-se a importância de estudos epidemiológicos recentes sobre essa enfermidade, porém, a maioria dos trabalhos publicados sobre esse assunto estão desatualizados. Isso mostra que há necessidade de avaliar os dados designados a esse estudo. Desse modo, a abordagem desse estudo teve como objetivo descrever o perfil epidemiológico dos casos de meningite, notificados e confirmados, entre as crianças de o a I2 anos e dos pré- 
adolescentes de I2 a I4 anos do estado de São Paulo, no período de 2016 a 2020, incluindo sexo e evolução, além de comparar os grupos etários analisados.

\section{OBJETIVOS}

Geral: Analisar a prevalência dos casos de meningite, por evolução, no estado de São Paulo, durante o período de 2016 a 2020, em crianças de o a I2 anos e pré-adolescentes de I2 a I4 anos. Específicos: Mensurar as variáveis sexo, evolução e comparar a prevalência entre as faixas etárias incluídas no estudo - Menor que I ano; de I-4; de 5-9 e de IO-I4 - dos casos de meningite.

\section{METODOLOGIA}

Trata-se de um estudo epidemiológico de caráter observacional, transversal, descritivo, com abordagem quantitativa e de prevalência, realizado através do Sistema de Informação de Agravos de Notificações (SINAN). O acesso à plataforma do SINAN foi realizado a partir do DATASUS, base de dados secundários, através do item "Epidemiológicas e Morbidade", seção de "Doenças e Agravos de Notificação - 2007 em diante (SINAN)".

Diante disso, foram computados para análise, o número total de casos de meningite em crianças e pré-adolescentes, na faixa etária de o a I4 anos, no estado de São Paulo, entre os anos de 2016 a 2020, notificados e registrados no TABNET.

Foram considerados como critérios de inclusão, estar notificado no SINAN e possuir caso confirmado de meningite, com base nas normas do Sistema de Vigilância Epidemiológica do Ministério da Saúde. As variáveis analisadas foram: gênero (masculino; feminino), faixa etária (Menor que I ano; de I-4; de 5-9 e de Io-I4) e evolução (alta; óbito por meningite; óbito por outra causa).

Foram excluídos da pesquisa os casos de meningite não notificados e registrados no SINAN, os casos registrados nos anos anteriores a Janeiro de 2016 e nos anos posteriores a Dezembro de 2020, os casos de crianças e pré-adolescentes com idade superior a I4 anos e os casos registrados em estados diferentes de São Paulo. Além disso, foram excluídos os casos considerados "ignorados" ou "em branco" para as variáveis analisadas. 
A análise de dados foi realizada e organizada em tabelas descritivas e analíticas, a partir do software Microsoft Excel ${ }^{\circledR}$, contendo frequências absolutas e relativas para comparação das variáveis analisadas em cada período.

No que concerne às normas éticas de pesquisa, o estudo fundamentou-se na Resolução no 5 Io do CNS, de 7 de abril de 2016, artigo I, incisos I, II, III e V, que isenta pesquisas que utilizam informações de acesso e domínio público e pesquisas com bancos de dados, cujas informações são agregadas, sem possibilidade de identificação pessoal, de registro e avaliação pelo Comitê de Ética em Pesquisa da Comissão Nacional de Ética em Pesquisa - sistema CEP/CONEP.

\section{RESULTADOS}

Foram observados 15.332 casos de meningite em crianças e pré-adolescentes durante o período avaliado. Houve uma prevalência no ano de 2018, com 4.234 (Tabela I) casos, e uma menor em 2020, com apenas 682 casos. Verificou-se um predomínio de meningite na faixa etária de I a 4 anos de idade com 6074 (Gráfico I) casos e um menor número de casos pertencente às crianças entre ro e $\mathrm{I} 4$ anos de idade, as quais representam 1387 indivíduos acometidos por essa enfermidade.

Quanto a diferenciação de sexo entre os pacientes com meningite que obtiveram alta, verificou-se que 8.755 destes eram do sexo masculino, sendo assim predominantes. Em seguida foi observada a quantidade absoluta de crianças e pré-adolescentes do sexo feminino, os quais constituíram um número de 6.025 dos casos (Tabela I).

É importante salientar que algumas notificações não apresentavam o registro do sexo, sendo assim 552 pacientes foram ignorados.

Ademais, foi observado durante a pesquisa que o número de indivíduos do sexo masculino representou uma taxa de mortalidade de $16,37 \%$, já o sexo feminino foi de $12,65 \%$. Consoante a isso, o número de óbitos por outra causa segue o mesmo padrão uma vez que os homens representam a maioria dos óbitos.

Portanto, observou-se uma predominância dos casos de meningite no Estado de São Paulo em crianças na faixa etária de i a 4 anos, no ano de 2018 com maioria sendo do sexo masculino. Dessa forma, obteve-se que a faixa etária de i a 4 anos representou $39,61 \%$ dos 
casos de meningite notificados durante esses 5 anos observados na pesquisa. Além disso, a taxa de pacientes que obtiveram alta foi $96,39 \%$ e a taxa de mortalidade apresentada no período citado foi de $2,9 \%$.

Gráfico I. Avaliação da taxa de óbitos por meningite, óbitos por outras causas, altas e a prevalência da população estudada, de acordo com a faixa etária.

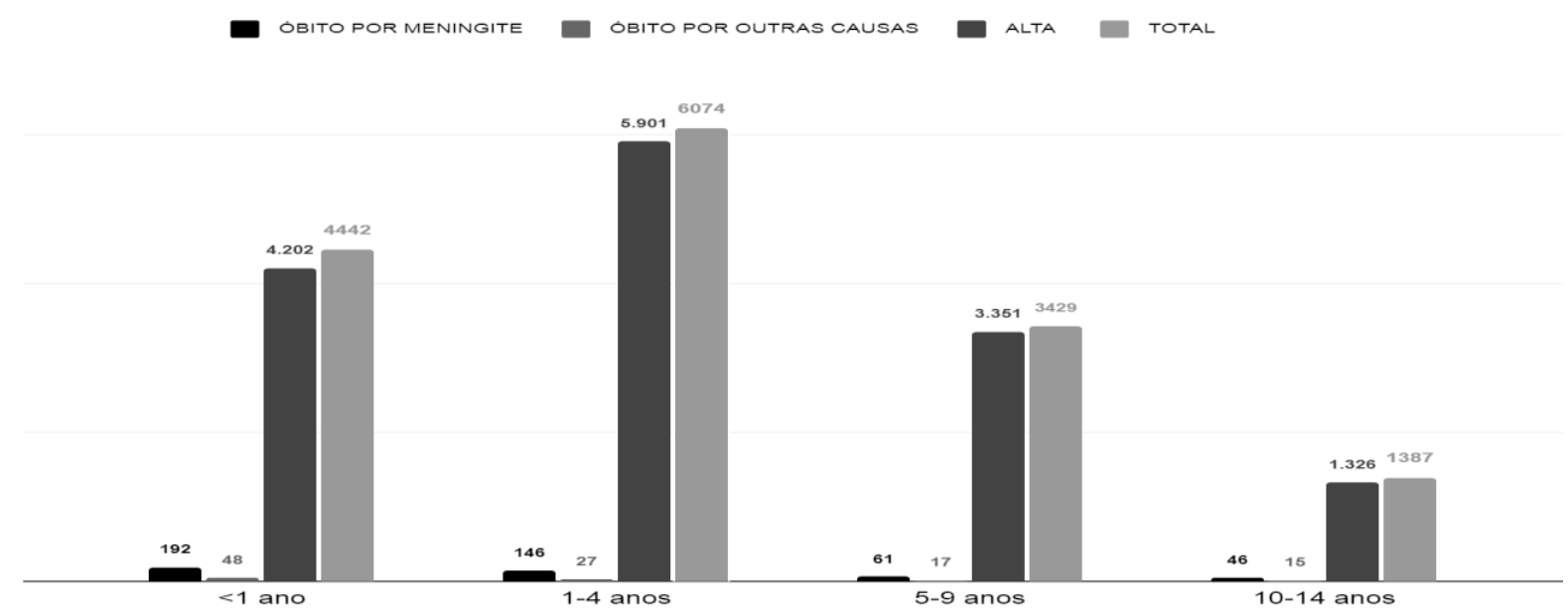

Fonte: Ministério da Saúde/SVS - Sistema de Informação de Agravos de Notificação; 202i. .

Tabela r. Comparação do número de casos confirmados de Meningite a partir dos anos de maior e menor prevalência e do sexo.

\begin{tabular}{cccc} 
Ano & & n & $(\%)$ \\
\hline & $\mathbf{2 0 1 8}$ & 4.234 & $27,6 \%$ \\
& 2020 & 682 & $4,4 \%$
\end{tabular}

\begin{tabular}{lccc} 
Sexo & & $\mathbf{n}$ & $(\%)$ \\
\hline & Maculino & 8.755 & $57,1 \%$ \\
& Feminino & 6.025 & $39,3 \%$
\end{tabular}

Fonte: Ministério da Saúde/SVS - Sistema de Informação de Agravos de Notificação; 202I.

\section{DISCUSSÃO}

A meningite é uma doença infecciosa que acomete principalmente crianças de o a 12 anos (HASBUN et al., 2019), crianças segundo Estatuto da Criança e do Adolescente (ECA), e pré-adolescentes de I2 a I4 anos. Dessa forma, considerando os dados epidemiológicos 
dispostos nos resultados, a prevalência observada nos indivíduos dessa população é na faixa etária de I a 4 anos, o que pode ser explicado pela baixa imunidade dessa população (MAGALHÃES; SANTOS, 2018).

Conforme afirmado por Santos (202I) em seu estudo epidemiológico, a meningite obteve um predomínio significativo em crianças quando comparado aos indivíduos de outras faixas etárias e embora seja letal, a maior parte dos agentes etiológicos bacterianos dessa enfermidade podem ser evitados por meio da vacinação .

Um estudo retrospectivo da meningite meningocócica (SOUZA, 2021) mostrou que essa doença pode ocasionar até 500 mortes por ano. Diante disso, foi observado que as taxas de óbito por meningite na população citada representaram 2,9\% dos casos confirmados por evolução, segundo o primeiro sintoma, durante os anos de 2016 a 2020. Logo, com base nesse dado, pode-se afirmar que a meningite não é uma das principais causas de mortalidade na população pediátrica. Além da avaliação do número de óbitos, foi realizado uma avaliação no quesito óbitos por sexo e concluiu-se que o sexo masculino foi o mais afetado pela meningite, representando $56,4 \%$ dos pacientes acometidos por essa enfermidade no período avaliado.

\section{Gráfico 2: Percentual de óbitos por Meningite.}

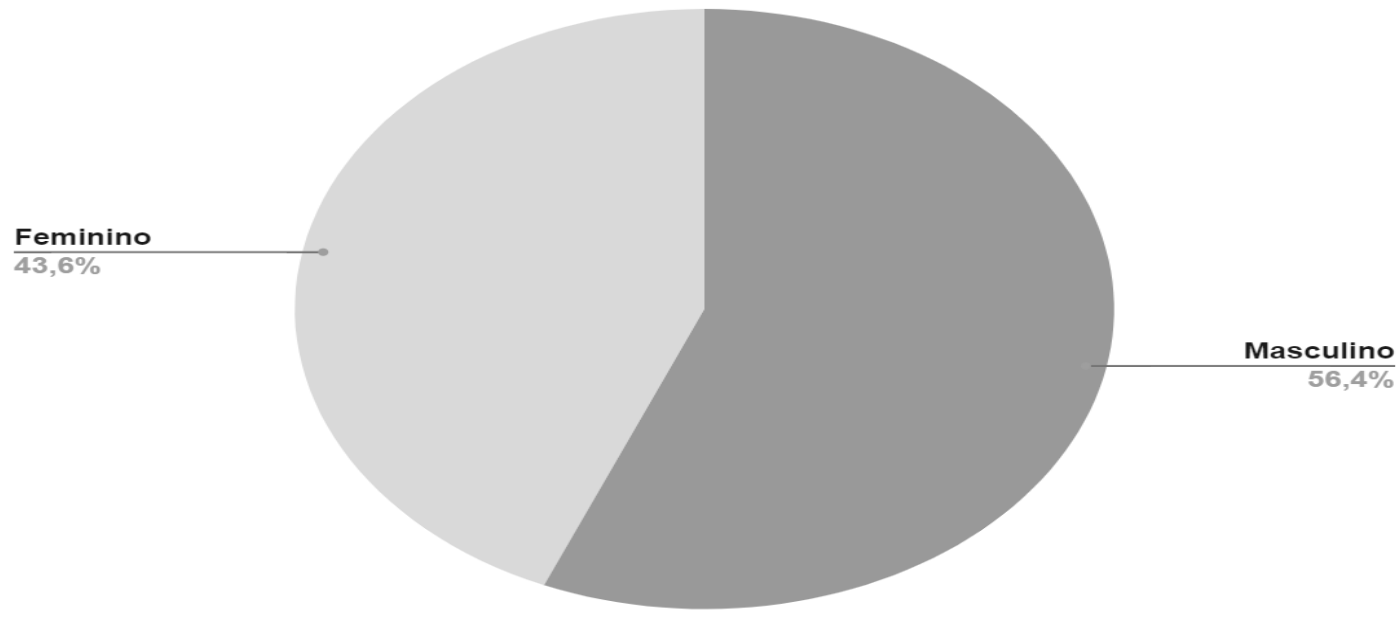

Fonte: :Ministério da Saúde/SVS - Sistema de Informação de Agravos de Notificação - SINAN Net, 202I 
Ademais, ao observar-se um panorama da atualidade (MAGALHÃES; SANTOS, 2018) notou-se um alto índice de internamento hospitalar por meningite, sendo este de $80 \%$. Outrossim, observou-se (gráfico I) um elevado índice de alta hospitalar que esteve presente em 14.780 casos avaliados no período, o que corresponde a $96,39 \%$ dos pacientes estudados nessa pesquisa. Logo, esse aumento na dispensa hospitalar se deve a efetividade do tratamento da meningite, o qual tem sido realizado de forma imediata e específica, respeitando e atendendo às especificidades das diferentes etiologias dessa doença.

Consoante ao estudo de (BEREZIN et al., 2002), este estudo conclui a necessidade da adoção de medidas profiláticas para o combate dessa enfermidade por parte da comunidade e das esferas governamentais de poder e saúde, como por exemplo a vacinação e o diagnóstico precoce. Logo, pode-se citar higienização e ventilação do ambiente, higienização correta das mãos e cuidado com os alimentos que serão utilizados para a alimentação. Além dessas medidas, a notificação compulsória se faz necessária quando se trata da doença.

\section{CONCLUSÃO}

A meningite é uma enfermidade que causa a diminuição da qualidade de vida do indivíduo acometido por essa doença, portanto, deve ser tratada de forma imediata e eficiente. Durante a realização desse estudo, o qual tomou como cenário base o Estado de São Paulo, durante os anos de 2016 a 2020, foi observado que a Meningite está mais presente na população de crianças entre i a 4 anos de idade uma vez que essa faixa etária representou $39,61 \%$ dos casos notificados. Ademais, é frequente nos indivíduos do sexo masculino, os quais compõem a maior taxa de óbito ocasionado por essa doença.

Por fim, a taxa de indivíduos que obtiveram alta foi de $96,39 \%$ demonstrando que embora a meningite seja uma doença de rápida evolução, houve uma taxa promissora de pacientes que tiveram uma recuperação eficiente e, posteriormente, liberação do ambiente hospitalar.

É de grande importância ressaltar que o correto preenchimento dos prontuários e fichas de notificação deve ser realizado com atenção e fidedignidade pelos profissionais da saúde, pois a partir desses dados poderão surgir significativos resultados de pesquisas, nos 
quais o próprio governo poderá se basear e se planejar para uma tomada de decisão mais efetiva em prol da população.

\section{REFERÊNCIAS}

BEREZIN, E. N. et al. Meningite pneumocócica na infância: características clínicas, sorotipos mais prevalentes e prognóstico. Jornal de Pediatria: Sociedade Brasileira de Pediatria, Rio de Janeiro, v. 78, ed. I, p. 19 - 23, 2002.

BRASIL. Vigilância Epidemiológica. Investigação de surto comunitário de doença meningocócica no Município de São Paulo, julho de 2007. Revista de Saúde Pública, v. 4I, n. 5, p. $873-78,2007$.

BRASIL. Ministério da Saúde. Gabinete do Ministro. Portaria no 204, de 17 de fevereiro de 2016.Define a Lista Nacional de Notificação Compulsória de doenças, agravos e eventos de saúde pública nos serviços de saúde públicos e privados em todo o território nacional, nos termos do anexo, e dá outras providências. Diário Oficial da União, Brasília, DF, i7 de fev. 2016.

BRASIL. Ministério da Saúde. Guia de Vigilância Epidemiológica 7.Ed. Normas e manuais técnicos. Brasília, 2009. Cad.I2. Disponível em: http://bvsms.saude.gov.br/bvs/publicacoes/guia_vigilancia_epidemiologica_7ed.pdf.

Acesso em i9 de maio de 202I.

CONDINO-NETO, A. Susceptibilidade a infecções: imaturidade imunológica ou imunodeficiência?. Revista de Medicina , São Paulo, v. 93, ed. 2, p. 78-82, 2014. https://doi.org/ıo.II6o6/issn.1679-9836.v93i2p78-82.

EMMERICK, I. C. M. et al. Estimativas corrigidas de casos de meningite, Brasil 2008-2009. Revista Epidemiologia e Serviços de Saúde, Brasília, v. 23, ed. 2, p. 215-226, 2014.

FERREIRA, J. H. S et al. TENDÊNCIA E ASPECTOS EPIDEMIOLÓGICOS DAS MENINGITES BACTERIANAS EM CRIANÇAS. Revista de Enfermagem: UFPE ONLINE. v,9, ed. 7, p. 8534-4I, 2015.

HASBUN, R. et al. Epidemiology of Meningitis and Encephalitis in Infants and Children in the United States, 2011-2014 The Pediatric Infectious Disease Journal.v. 38, ed.I, p.37-4I, 2019.

IVERSSON, L. B. Aspectos epidemiológicos da meningite meningocócica no município de São Paulo (Brasil), no período de 1968 a 1974. Revista de Saúde Pública, São Paulo, v. ıo, ed. I, p. I - 16, 1976. DOI https://doi.org/ro.1590/Soo34-89101976oooroooor. Disponível em: 
https://www.scielo.br/j/rsp/a/sLDTdNNzDsDYLp4VX6hd8CD/?lang=pt. Acesso em: I9 maio 202I.

MAGALHÃES ， R. S.; SANTOS, M. S. PERFIL EPIDEMIOLÓGICO DA MENINGITE BACTERIANA NO MUNICÍPIO DE VITÓRIA DA CONQUISTA BAHIA, NO PERÍODO DE 2008 A 2015. Revista de Ciências Médicas e Biológicas, Salvador, v. 17, ed. I, p. 33-39, 2018.

MORAES, J. C.; BARATA, R. B. A doença meningocócica em São Paulo, Brasil, no século $\mathrm{XX}$ : características epidemiológicas. Caderno de Saúde Pública, Rio de Janeiro, v. 2I, ed. 5 , p. 1458 -I47I, 2005 .

NESTI, M. M.; GOLDBAUM, M. As creches e pré-escolas e as doenças transmissíveis. Jornal de Pediatria: Sociedade Brasileira de Pediatria, Rio de Janeiro, v. 83, ed. 4, p. 299-312, 2007 .

OLIVEIRA, A; SIMÃO, A.C; NASCIMENTO , L.F.C. Perfil dos casos de meningite em serviço de pediatria. Revista paulista de pediatria , [s. l.], v. 22, ed. 22, p. 89-94, 2004. Disponível em: https://pesquisa.bvsalud.org/portal/resource/pt/ses-I4949. Acesso em: I9 maio 2021.

ROCHA, G. J. N.; COSTA, G. M. A.; SILVA, S. A. A. Análise transversal da incidência de infecção meningocócica em crianças e adultos de I a 59 anos em Sergipe comparativamente com o Brasil, nos anos de 2008 a 2017. Brazilian Journal of health Review, Curitiba, v. 2, ed. 4, p. 2677-2694, 2019.

RODRIGUES, E. M. B. MENINGITE: PERFIL EPIDEMIOLÓGICO DA DOENÇA NO BRASIL NOS ANOS DE 2007 A 2013. Orientador: Bruno Silva Milagres. 2015. I6 p. Trabalho de Conclusão de Curso (Bacharelado em Biomedicina) - Centro Universitário de Brasília, Brasília, 2015.

SANTOS, J. C. et al. MENINGITE NA INFÂNCIA: UMA ANÁlisE DAS INTERNAÇÕES HOSPITALARES NO BRASIL. Revista Científica da Escola Estadual de Saúde Pública de Goiás *Candido Santiago*, Goiás, v. 7, 202I.

SOUZA, E. F. M. et al. Análise Do Perfil Epidemiológico De Meningite Na Região Metropolitana De Belém-Pa Em Um Período De ıo Anos (2010 a 2019). Revista de Patologia do Tocantins, v. 7, n. 3, p. II-I7, 2020. 
SUGAWARA, E. e NIKAIDO, H.. Properties of AdeABC and AdeIJK efflux systems of Acinetobacter baumannii compared with those of the AcrAB-TolC system of Escherichia coli. Antimicrobial Agents and Chemotherapy, v. 58, n. 12, p. 7250-7257, 2014.

TAKADA, Shimon et al. Meningococcemia in Adults: A Review of the Literature. Internal Medicine , [s. l.], p. 567-572, 2016. DOI 10.2169/internalmedicine.55.3272. Disponível em: https://www.jstage.jst.go.jp/article/internalmedicine/55/6/55_55.3272/_pdf/-char/en.

Acesso em: 19 maio 2021. 